\title{
Preliminary study of an exercise programme for reducing fatigue and improving sleep among long-term haemodialysis patients
}

Radha $\frac{\text { Maniam }^{1} \text {, MNSc, Pathmawathi Subramanian }}{1}$, DHSci, MEd, Surindar Kaur Surat Singh ${ }^{1}$, MSN,

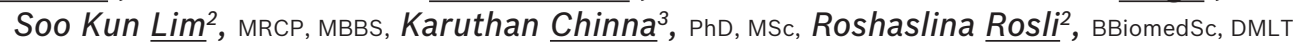

INTRODUCTION Fatigue and quality of sleep are the main factors that contribute to a poor quality of life among patients on long-term haemodialysis. Studies have also emphasised the importance of exercise for improving the wellbeing of dialysis patients. This study aimed to determine the effectiveness of a predialysis low-to-moderate-intensity exercise programme for reducing fatigue and improving sleep disorders among long-term haemodialysis patients.

METHODS In this quasi-experimental study, an exercise programme was conducted three times a week for 12 weeks before long-term haemodialysis patients underwent dialysis at two centres. The patients were categorised into either the exercise group $(n=28)$ or control group $(n=27)$. The latter was asked to maintain their current lifestyles. Assessments of fatigue and sleep disorder levels were performed for both groups using self-reported questionnaires at baseline and after intervention. The patients' perception of the exercise programme was also determined using self-reported questionnaires. RESULTS Paired sample $t$-test indicated improvements in fatigue level in the exercise group (mean fatigue score: posttreatment $40.5 \pm 7.9$ vs. pre-treatment $30.0 \pm 10.9$ ). Improvements in sleep disorders were also observed in the exercise group (mean score: post-treatment $7.6 \pm 3.3$ vs. pre-treatment $10.1 \pm 3.8$ ). However, sleep quality deteriorated in the control group (mean score: post-treatment $10.7 \pm 2.9$ vs. pre-treatment $9.3 \pm 2.9$ )

CONCLUSION Simple low-to-moderate-intensity exercise is effective for improving fatigue, sleep disorders and the overall quality of life among haemodialysis patients.

Keywords: exercise, fatigue, haemodialysis, sleep disorder

\section{INTRODUCTION}

Patients with end-stage renal disease develop many complications due to the multisystem effects of renal failure and its treatment. Among these, fatigue is often considered by patients to be the most bothersome symptom, as it deprives them of a normal life. Studies have shown that fatigue is significantly associated with the presence of sleep problems, poor physical health status and depression among patients receiving haemodialysis. ${ }^{(1-4)}$ Cupisiti et al reported that inactivity and reduced physical functioning and performance among patients with renal failure are caused by fatigue, which in turn leads to a sedentary lifestyle. ${ }^{(5)}$ According to Wong and Ong, fatigue not only impacts the quality of life but also increases the risk of cardiac disease in these patients, as it fosters a sedentary lifestyle. ${ }^{(6)}$

Over the years, evidence from published studies has emphasised the importance of exercise for improving the wellbeing of dialysis patients, even as several studies have suggested that physical inactivity in dialysis patients is often defined by a lack of health-enhancing moderate-to-vigorous activity. ${ }^{(2,5,7-9)}$ Chang et al reported that improvements in physical fitness and psychological function, which translated to improved quality of life, were noted in patients with chronic kidney disease who exercised. ${ }^{(10)}$ Others have found that exercise programmes of mild-to-moderate intensity improved fatigue and quality of sleep, the two main factors that are believed to contribute to poor quality of life among patients with renal failure..$^{(11-14)}$
Notwithstanding the above findings, the prescription of exercise for the rehabilitation of dialysis patients in Malaysia is still in its infancy. We undertook the present study to gain insights into the effects of exercise on fatigue and quality of sleep among dialysis patients in the country, and to ascertain its ability to enhance patients' quality of life. We aimed to determine the effectiveness of a predialysis low-to-moderate-intensity exercise programme for reducing fatigue and improving sleep disorders among long-term haemodialysis patients, as well as gauge patients' perception of the programme.

\section{METHODS}

A quasi-experimental, non-equivalent groups pretest and posttest design was used to study the effectiveness of a simple low-tomoderate-intensity exercise programme for the management of fatigue and sleep disorders in long-term haemodialysis patients. Participants were recruited from two haemodialysis centres in Kuala Lumpur, Malaysia, and voluntarily enrolled into the exercise programme. Inclusion criteria were patients aged $\geq 18$ years, a minimum duration of six months of regular haemodialysis with dialysis efficiency $(\mathrm{Kt} / \mathrm{V})$ results $>1.0$, controlled systolic blood pressure (range 110-180 mmHg), no evidence of shortness of breath on rest, haemoglobin level $\geq 9.5 \mathrm{~g} / \mathrm{dL}$, and fatigue score $<43$ based on the Functional Assessment of Chronic Illness Therapy (FACIT)-fatigue scale ${ }^{(11)}$ Exclusion criteria were patients

${ }^{1}$ Department of Nursing Science, ${ }^{2}$ Department of Medicine, ${ }^{3}$ Department of Social and Preventive Medicine, Faculty of Medicine, University of Malaya, Kuala Lumpur, Malaysia Correspondence: Dr Pathmawathi Subramanian, Senior Lecturer, Department of Nursing Science, Faculty of Medicine, University of Malaya, 50603 Kuala Lumpur, Malaysia. pathmawathi@um.edu.my 
with chronic obstructive pulmonary disease, symptomatic cardiac disease, active infection and severe musculoskeletal disorders. Participants who were already on regular physical fitness regimens were also excluded.

A total of 102 patients undergoing long-term haemodialysis treatment at two haemodialysis centres during the study period were invited to take part in the study. Of these, only 73 patients volunteered to participate. Following the application of the inclusion and exclusion criteria, the number of eligible potential participants dropped to 55. Among these, 28 patients agreed to participate in the exercise programme and the remaining 27 patients were placed in the control group. Ethics approval was obtained from the hospital's ethics committee. All patients were provided with information on the exercise programme and consent was obtained from all patients who agreed to participate in the study.

Assessments of fatigue and sleep disorder levels were conducted using self-reported questionnaires at baseline and post intervention for both patient groups. The exercise programme was conducted before dialysis three times a week for 12 weeks. The control group was asked to maintain their current lifestyle during that period. For patients in the exercise group, their perception of the exercise programme was also determined using self-reported questionnaires after the intervention.

Patients in the exercise group were assigned regular flexibility and strengthening exercises for about 30-40 min prior to each dialysis session, with three sessions arranged every week. The exercise programme was based on the book Exercise: A Guide for People on Dialysis by Patricia Painter. ${ }^{(7)}$ During the first few sessions, a qualified physical trainer was present to instruct patients on the correct techniques, and to increase their confidence and competence. Each exercise session commenced with five repetitions of deep breathing exercises, where patients were instructed to inhale through the nose, retain their breath and then exhale slowly. This was followed by flexibility exercises, where patients were required to gently stretch their muscles until light tension was felt on the concentrated muscles for $10 \mathrm{~s}$. Each step was repeated five times. After the flexibility exercises, strength exercises were performed for the next 30 min. Patients were required to perform strength exercises until they achieved a level of 12-13 (60\% work effort), based on the Rated Perceived Exertion (RPE) scale. ${ }^{(12)}$ The intensity of the exercise was adjusted according to individual capabilities. Patients were allowed to rest when they felt tired. Every session ended with relaxation exercises, where patients were instructed to sit still and close their eyes while breathing deeply for five times. Music was played during the exercise session to create a calm and relaxing environment. No injury or decline in health condition related to the exercise programme was noted among patients throughout the study period.

Fatigue levels experienced in the seven days preceding the exercise programme were measured using the Malay version of the self-reported FACIT-fatigue scale, a 13-item questionnaire with a five-point Likert scale and scores ranging from $0-52$, with a lower score indicating a higher level of fatigue. Although the cutoff score was 43, patients who scored lower than 43 were included in our study, as the lower score was taken to represent higher fatigue levels in our patients. ${ }^{(13)}$ This tool assesses multiple aspects of fatigue and its impact on daily life, and included questions on tiredness, weakness, difficulty in carrying out one's usual activities and measurement of one's degree of fatigue.

Sleep disorders were assessed using the Malay version of the Pittsburgh Sleep Quality Index (PSQI). ${ }^{(14)}$ A total of 19 selfreported questions assessed sleep quality, frequency and severity of specific sleep-related problems during the previous month, and categorised patients as either good or poor sleepers. The 19 items were grouped into seven components (sleep quality, sleep latency, sleep duration, habitual sleep efficiency, sleep disturbance, use of sleep medications and daytime dysfunctions) and scored from $0-3(0=$ very good; 1 = fairly good; 2 = fairly bad; 3 = very bad $)$. The scores were added to yield a global PSQI score in the range 0-21, with a higher score indicating poorer sleep quality. A global PSQI score $\geq 5$ was suggestive of significant sleep disorders and indicated that the person was a poor sleeper.

A questionnaire was also developed to determine patients' perception of the exercise programme based on the selfdetermination theory, ${ }^{(15)}$ which emphasises the creation of an environment that provides autonomy, competence and relatedness to achieve intrinsic motivation. Questions that assessed the three components of intrinsic motivation were created to examine patients' changed behaviour following intervention. This was a ten-item, self-reported questionnaire with a three-point Likert scale $(0=$ not true; $1=$ sometimes true; $2=$ true $)$.

Reliability tests were conducted for the aforementioned three instruments. Cronbach's alpha was 0.72 for the Malay version of the FACIT-fatigue scale, while that for the Malay version of the PSQI was 0.81. For our self-developed questionnaire on patients' perception of the exercise programme, which was tested on eight patients, Cronbach's alpha was 0.88, thus confirming its internal consistency. For validity, the contents of the three questionnaires were validated by a clinical nephrologist and a renal clinical nurse lecturer.

Data was analysed using the Statistical Package for the Social Sciences version 16 (SPSS Inc, Chicago, IL, USA). Descriptive statistics (in terms of mean \pm standard deviation and frequency) were calculated for variables such as age, gender, marital status, education, employment, duration on haemodialysis, fatigue, sleep disorders and perception of exercise. Independent $t$-test was used to compare patient characteristics between the exercise and control groups. Paired sample $t$-test was used to compare the changes within the groups. Cohen's effect size was calculated to determine the effectiveness of the exercise programme. A p-value $<0.05$ was considered to be statistically significant. Cronbach's alpha reliability coefficient was used to assess the internal consistency of the study questionnaires, with acceptance value set at 0.7 .

\section{RESULTS}

A total of 55 patients agreed to participate in the study - 28 patients were included in the exercise group and 27 in the control group. 
Table I. Demographic characteristic of the participants.

\begin{tabular}{|c|c|c|c|c|}
\hline \multirow[t]{2}{*}{ Variable } & \multicolumn{3}{|c|}{ No. (\%) } & \multirow[t]{2}{*}{ p-value } \\
\hline & $\begin{array}{l}\text { Overall } \\
(n=47)\end{array}$ & $\begin{array}{l}\text { Exercise group } \\
(n=20)\end{array}$ & $\begin{array}{l}\text { Control group } \\
(n=27)\end{array}$ & \\
\hline Gender & & & & 0.58 \\
\hline Male & $26(55.3)$ & $12(60.0)$ & $14(51.9)$ & \\
\hline Female & $21(44.7)$ & $8(40.0)$ & $13(48.1)$ & \\
\hline Marital status & & & & 0.63 \\
\hline Married & $41(87.2)$ & $18(90.0)$ & $23(85.2)$ & \\
\hline Single & $6(12.8)$ & $2(10.0)$ & $4(14.8)$ & \\
\hline Education level & & & & 0.17 \\
\hline Primary & $26(55.3)$ & $9(45.0)$ & $17(63.0)$ & \\
\hline Secondary & $19(40.4)$ & $9(45.0)$ & $10(37.0)$ & \\
\hline Tertiary & $2(4.3)$ & $2(10.0)$ & - & \\
\hline Yes & $6(12.8)$ & $3(15.0)$ & $3(11.1)$ & \\
\hline No & $41(87.2)$ & $17(85.0)$ & $24(88.9)$ & \\
\hline Duration of haemodialysis & & & & 0.05 \\
\hline $6 \mathrm{mths}$ to $<5$ yrs & $14(29.8)$ & $9(45.0)$ & $5(18.5)$ & \\
\hline$\geq 5$ yrs & $33(70.2)$ & $11(55.0)$ & $22(81.5)$ & \\
\hline
\end{tabular}

*Data is presented as mean \pm standard deviation (range). ${ }^{\dagger} \mathrm{p}$-value was calculated using independent sample $t$-test.

Table II. Effects of exercise on fatigue score.

\begin{tabular}{|c|c|c|c|c|c|c|c|}
\hline \multirow[t]{2}{*}{ Group } & \multicolumn{3}{|c|}{ Fatigue score* } & \multirow[t]{2}{*}{$\mathbf{t}$} & \multirow[t]{2}{*}{ df } & \multirow[t]{2}{*}{ p-value } & \multirow{2}{*}{$\begin{array}{l}\text { Effect } \\
\text { size }\end{array}$} \\
\hline & Pre-treatment & Post-treatment & Mean difference & & & & \\
\hline Exercise & $30.0 \pm 10.9$ & $40.5 \pm 7.9$ & $-10.6 \pm 9.2$ & -5.2 & 19 & $<0.001$ & -1.1 \\
\hline
\end{tabular}

* Data is presented as mean \pm standard deviation.

However, among the patients in the exercise group, four withdrew from the exercise programme at the initial stage and four were excluded from the study - one patient was excluded due to eye infection, while three were excluded due to transportation-related difficulties that affected attendance. Finally, 47 patients completed the study - 20 (71\%) patients in the exercise group and $27(100 \%)$ in the control group.

The mean age of the patients was $57.1 \pm 8.8$ (range 40-74) years (Table I). The majority of patients $(n=41,87.2 \%)$ were unemployed. With respect to gender, there was a near-equal distribution of men $(n=26)$ and women $(n=21)$. Most of the patients $(n=33,70.2 \%)$ had received haemodialysis for five years or more.

Table Il shows the effect of exercise on fatigue level. The mean fatigue score at baseline among all 47 patients was $32.3 \pm 9.4$. The control group had higher mean fatigue scores at baseline than the exercise group $(34.1 \pm 8.3$ vs. $30.0 \pm 10.9$, Table II), indicating that, prior to treatment, patients in the exercise group were more fatigued than those in the control group. The lowest fatigue score among patients in the exercise group was 10, which further reinforced the fact that patients in this group were more fatigued. However, independent sample $t$-test of baseline fatigue scores showed no statistically significant difference in the fatigue levels of patients in the two groups ( $p>0.05$ ).

Data from the paired sample $t$-test indicated that, in the exercise group, mean post-treatment fatigue scores were higher than the pre-treatment levels $(40.5 \pm 7.9$ vs. $30.0 \pm 10.9)$. The minimum range for fatigue scores in the exercise group increased

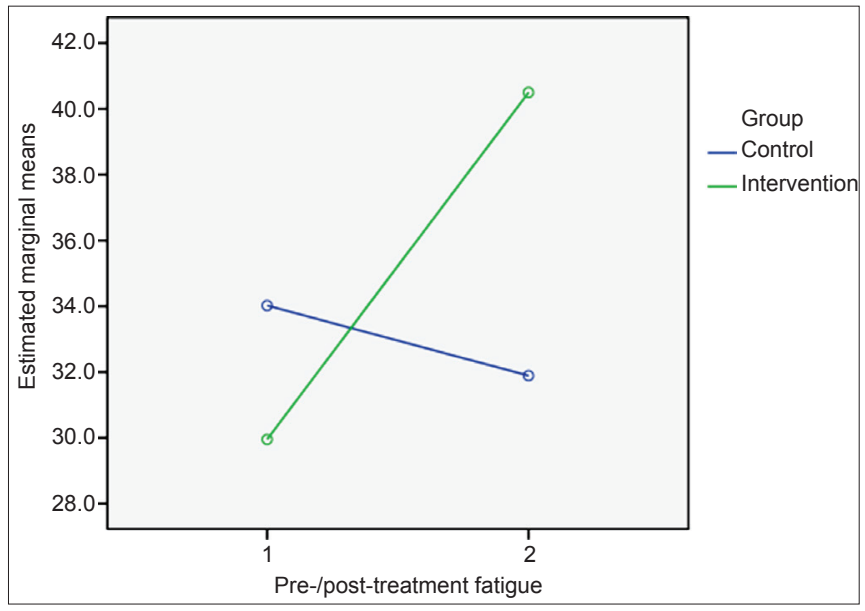

Fig. 1 Effects of exercise on fatigue scores.

from 10 to 22, reflecting an improvement in post-treatment fatigue levels. In the control group, however, the mean fatigue score decreased from $34.1 \pm 8.3$ pre-treatment to $31.9 \pm 9.2$ post-treatment, indicating that fatigue levels worsened in these patients during the study period (Table II \& Fig. 1). The calculated effect size for the exercise group $(d=-1.1)$ indicated a large effect size, establishing that there was improvement in the fatigue level following exercise. Meanwhile, in the control group, a moderate effect size $(d=0.5)$ was evident.

On the self-reported FACIT-fatigue scale, the highest mean scores were seen for the items 'need to sleep during daytime' 
Table III. Effects of exercise on the global Pittsburgh Sleep Quality Index (PSQI) score.

\begin{tabular}{|c|c|c|c|c|c|c|c|}
\hline \multirow[t]{2}{*}{ Group } & \multicolumn{3}{|c|}{ PSQI score* } & \multirow[t]{2}{*}{$\mathbf{t}$} & \multirow[t]{2}{*}{ df } & \multirow[t]{2}{*}{ p-value } & \multirow{2}{*}{$\begin{array}{c}\text { Effect } \\
\text { size }\end{array}$} \\
\hline & Pre-treatment & Post-treatment & Mean difference & & & & \\
\hline Exercise & $10.1 \pm 3.8$ & $7.6 \pm 3.3$ & $2.5 \pm 2.4$ & 4.6 & 19 & $<0.001$ & 1.0 \\
\hline Control & $9.3 \pm 2.9$ & $10.7 \pm 2.9$ & $-1.4 \pm 1.8$ & -4.0 & 26 & $<0.001$ & -0.8 \\
\hline
\end{tabular}

* Data is presented as mean \pm standard deviation.

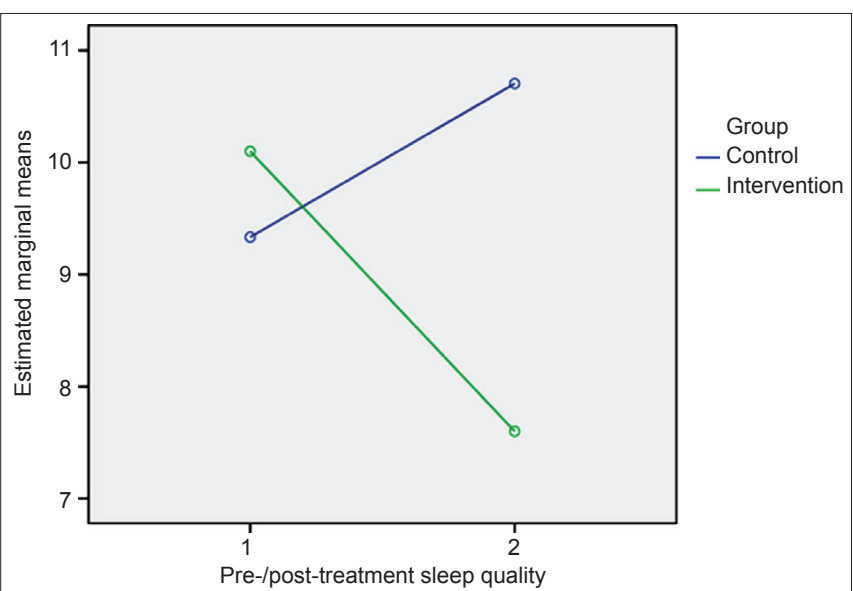

Fig. 2 Effects of exercise on the Pittsburgh Sleep Quality Index score.

$(2.6 \pm 3.2)$, 'feeling fatigued' $(2.0 \pm 1.4)$, 'feeling tired' $(1.9 \pm 1.2)$ and 'feeling weak' $(1.8 \pm 1.3)$. Lower mean scores were seen for the items 'need help in doing usual activities' $(0.9 \pm 1.1)$, 'have trouble starting' $(1.0 \pm 1.2)$ and 'finishing things' $(1.0 \pm 1.2)$. However, the item 'I have energy' also scored $2.0 \pm 0.6$, suggesting that patients were not severely affected by fatigue. Among the subscales, the mean score for the item 'I need to sleep during the day' decreased from $2.4 \pm 1.5$ pre-treatment to $1.1 \pm 1.1$ post-treatment. Reductions were also seen in all other items. There was improvement in the score for the item 'I have energy' from $2.1 \pm 0.6$ pre-treatment to $2.5 \pm 1.0$ post-treatment, and in other abilities to perform routine chores. The mean score for all items in the subscales showed deterioration in condition.

Table III shows the effect of exercise on the level of sleep quality. The mean global PSQI score at baseline among all patients was $9.7 \pm 3.1$, with $42(89.4 \%)$ patients rated as poor sleepers. The mean PSQI scores at baseline were higher in the exercise group than in the control group $(10.1 \pm 3.8$ vs. $9.3 \pm 2.9)$, indicating that patients in the exercise group had poorer quality of sleep prior to treatment. However, independent sample $t$-test of baseline PSQI scores showed no statistically significant difference in the quality of sleep of patients in the two groups ( $p>0.05)$.

Data from the paired sample $t$-test indicate that, in the exercise group, mean global PSQI scores were lower post-treatment than pre-treatment $(7.6 \pm 3.3$ vs. $10.1 \pm 3.8)$. In contrast, in the control group, the mean global PSQI score was higher post-treatment than pre-treatment $(10.7 \pm 2.9$ vs. $9.3 \pm 2.9)$, indicating a worsening of sleep disorders and deterioration of sleep quality in these patients over the study period (Table III \& Fig. 2). The calculated effect size for the exercise group $(d=1.0)$ indicated a large effect size, suggesting improvement in sleep disorders following exercise. Meanwhile, in the control group, the effect size was more moderate $(d=-0.8)$.
The subscale scores were separately analysed for the control and exercise groups. On the self-reported PSQI questionnaire, the highest mean score was seen for the component 'habitual sleep efficacy' $(1.9 \pm 0.2)$. When analysed separately, this item obtained the highest scores for both patient groups (median 3.0; interquartile range 0 ). Independent sample $t$-test and MannWhitney $U$ test of the PSQI scores indicated that there was no statistically significant difference in the global PSQI scores for any item between the two groups ( $p>0.05)$.

Although the majority of patients $(n=14,70 \%)$ in the exercise group continued to be considered poor sleepers based on the assessment of sleep quality, there was an increment in the number of patients ( $n=3,15 \%$ ) who were deemed to be good sleepers after the exercise programme. In the exercise group, all items on the self-reported PSQI questionnaire, except for the item 'habitual sleep efficacy', scored lower after the exercise programme, indicating improvement in sleep quality post-treatment. For the item 'habitual sleep efficacy', no difference was observed in the mean pre-treatment and post-treatment scores. However, in the control group, all items, except 'habitual sleep efficacy', showed an increase in mean scores at the end of the exercise programme, indicating deterioration in sleep quality during the study period. Similar to the exercise group, no difference was observed in the mean pre-treatment and post-treatment scores for control group patients as well.

In terms of patient perception of the exercise programme, the majority of patients in the exercise group $(n=11,55 \%)$ agreed that their quality of life improved following the exercise programme. All the patients $(n=20)$ felt that the exercises were easy to perform. They also indicated that they derived pleasure from exercising and were confident enough to perform the exercises at home. However, most patients preferred to exercise at the centres $(n=14)$, as they felt that it was more enjoyable $(n=20)$ and they were being cared for $(n=20)$. Even though all the patients agreed that it was important to exercise regularly, six patients did not see any value in the benefits of exercise.

\section{DISCUSSION}

In Malaysia, the prescription of exercise for the rehabilitation of patients on dialysis is still in its infancy. The purpose of the present study was to determine the effectiveness of a predialysis low-to-moderate-intensity exercise programme for improving the quality of life of patients on long-term haemodialysis, through the reduction of fatigue and improvement of sleep disorders.

Demographically, our study cohort was representative of the Malaysian dialysis population - the mean age and distribution of genders in our study are in agreement with those of a previous 
study ${ }^{(16)}$ conducted among this population. According to the 18th Report of the Malaysian Dialysis and Transplant Registry, the majority of dialysis patients in Malaysia were over 55 years old, and the male-to-female ratio had remained between $45 \%$ and $55 \%$ since 2001 , indicating that a near-equal number of men and women in the country undergo dialysis. ${ }^{(16)}$ In addition, the majority of our patients $(70.2 \%)$ had been receiving haemodialysis for five years or more and most $(87.2 \%)$ were unemployed, which increased their risk of leading a sedentary lifestyle.

Our study aimed to identify the outcome of two dependent variables - fatigue and sleep disorders - in long-term haemodialysis patients who participated in a simple exercise programme. To our knowledge, this is the only study that examined the outcome of a simple exercise programme, not involving vigorous exercises (such as aerobics, cycling or walking), in this patient population. Our exercise programme was planned to enable patients to achieve a work effort of at least $60 \%-80 \%$ based on the RPE scale. This was to ensure that patients would derive cardiovascular health benefits from the programme, as well as improvements in outcome, despite the simplicity of the exercises. ${ }^{(17)}$ As most dialysis providers in Malaysia are from the private sector and non-governmental organisations, proper gymnasiums or exercise equipment are not always available in these centres. ${ }^{(16)}$ Furthermore, attending nurses are often not qualified to teach patients to perform vigorous exercises. Given these factors, our exercise programme was designed to be simple so that it could be easily taught and guided by a group of selected nurses.

Our findings on fatigue among long-term haemodialysis patients as a consequence of renal failure and dialysis treatment are also in agreement with those of an earlier published study. ${ }^{(18)}$ In our study, patients in the exercise group experienced a higher level of fatigue prior to their participation in the exercise programme than those in the control group. This could be a contributing factor for their willingness to be part of the programme. However, we also noted that many patients whose quality of life was not severely affected by fatigue refused to exercise. These patients felt sufficiently energetic and capable of carrying out routine chores in spite of their need to sleep during the daytime in order to overcome tiredness and fatigue.

Olson highlighted that a person's condition may further deteriorate if no timely interventions were made to overcome fatigue. ${ }^{(19)}$ Similarly, Santhana et al reported that a lack of awareness and the acceptance of one's condition as the norm for ageing and disease progression were key reasons people on dialysis did not change their behaviour. ${ }^{(20)}$ As most patients in our study were over 55 years old and had been undergoing haemodialysis for five years or more, it was expected that their conditions would worsen over the years if no appropriate interventions were instituted to overcome fatigue. Hence, healthcare personnel play an important role in educating haemodialysis patients on the benefits of exercise, and in motivating and helping them to adopt a healthier lifestyle by engaging in exercise programmes that could help prevent further deterioration of their health condition.

As in the case of fatigue, we found that patients in the exercise group had more severe sleep disorders than those in the control group. Studies have reported a close relationship between fatigue and sleep disorders, suggesting that patients with higher levels of fatigue may also have poorer quality of sleep. ${ }^{(3,13)}$ Merlino et al reported that the foremost sleep disorders faced by dialysis patients with end-stage renal disease were initiating sleep at night and fewer hours of nighttime sleep, fitting the definition of insomnia. ${ }^{(21)}$ It is possible that insomnia could be related to the high incidence of daytime sleep in these patients. The majority of our patients reported the need to sleep during the day due to tiredness and fatigue, and this may be associated with difficulty in initiating sleep and having a good night's sleep. Reinforcing the cycle, reduced nighttime sleep could subsequently lead to more tiredness, weakness and fatigue, thus increasing their need for daytime naps and perpetuating poor nighttime sleep in these patients.

Insomnia in our patients could also be associated with variables such as age and anxiety levels. For instance, Merlino et al reported that age was associated with sleep disorders. ${ }^{(21)}$ Due to the older age of our cohort, it is possible that the prevalence of insomnia in our study was incidental to age. Studies by McCann and Boore ${ }^{(3)}$ and Sklar et $\mathrm{al}^{(22)}$ have reported high levels of depression among long-term haemodialysis patients, with factors such as enduring life with renal failure, long hours of haemodialysis and its complications, uncertainty about the future and financial issues being noted as the main causes of depression in these patients. Although there may have been a similar association between age and depression in our patients, this relationship was not investigated in our study.

The results of our study suggest that a simple flexibility and strengthening exercise programme, targeting a maximum work effort range of $60 \%-80 \%$, could improve fatigue and sleep quality among long-term haemodialysis patients. Many patients reported that they were better able to perform routine chores and felt more energetic post-treatment. Similarly, patients also indicated improved nighttime sleep post-treatment. These findings are likely interrelated, as patients, being less fatigued during the daytime, would have had a reduced need for daytime sleep and thus experienced improved nighttime sleep.

Similar results were reported by Yutkuran et al, ${ }^{(23)}$ Malagoni et al ${ }^{(24)}$ and Reza et al. ${ }^{(25)}$ However, these authors had employed different kinds of exercise programmes such as yoga-based exercises and aerobic training. Although earlier studies involving more vigorous exercise programmes generated positive outcomes similar to those of our study, increased muscular fatigue leading to high dropout rates was also reported. ${ }^{(23,26)}$ In van Vilsteren et al's study, older participants were reported to need a longer period to get used to vigorous exercises, and for that reason, require longer adjustment periods. ${ }^{(26)}$ In our study, vigorous exercises could aggravate fatigue and lead to other complications during exercise, as many of our haemodialysis patients also had coexisting conditions such as diabetes mellitus, cardiovascular disease and anaemia. A sedentary lifestyle was further reinforced by factors such as older age, unemployment status and lack of knowledge. In all likelihood, the introduction of vigorous activity to our cohort may prove to be not only a burden but also a demotivating factor to patients' 
willingness to enroll or continue in the exercise programme. Taking this into account, a less vigorous exercise programme that could be modified according to the patients' capabilities was thought to be more appropriate for our cohort.

We found that conducting the exercise programme on dialysis days prior to the dialysis sessions increased adherence to the programme among patients, indicating that patients were more willing to participate in exercises on these days. A low dropout rate was seen in our study, as $71 \%$ of patients completed the exercise programme. Cheema and Singh similarly reported that exercise training on non-dialysis days resulted in higher attrition rates when compared to dialysis days. ${ }^{(27)}$ Practical reasons for this include additional transportation arrangements, costs and time required for exercise training on non-dialysis days, in addition to patients perceiving exercise on dialysis days as a routine activity prior to the dialysis session. These results suggest that, when prescribing exercise for the rehabilitation of haemodialysis patients, clinicians and healthcare personnel should bear in mind that conducting exercise training on dialysis days would be more feasible, convenient, effective and time-saving, and may promote greater exercise adherence among patients.

Achieving intrinsic motivation is an integral part of introducing permanent change in patient behaviour, such that patients would begin to engage in exercise for reasons such as self-satisfaction rather than pressure or rewards. ${ }^{(28)}$ For exercise training to be truly meaningful, it is necessary that exercise ceases to be seen as a burden. To this end, the use of RPE was a success in our study, as it gave our patients the autonomy to control and modify the exercise regime according to their capabilities, and thus ensured that they did not feel pressured. However, although our patients considered regular exercise to be important and had the confidence to perform the exercises at home, many preferred the exercise sessions to be held at the dialysis centre. Also, we found that the 12-week exercise programme instituted in our study was not long enough to bring about permanent change in patient behaviour, as many patients did not value the benefits of exercise or wish to carry on with it at home after the study. These findings suggest a need for close monitoring and education of patients regarding the benefits of exercises so as to ensure that behavioural changes introduced as part of rehabilitation become permanent. Ryan and Deci found that patients enjoyed exercising in a group due to its increased socialising effects. ${ }^{(28)}$ Many patients in our study likewise indicated that they enjoyed the group sessions more, and felt that they were being cared for and more motivated in the company of their friends. This likely contributed to the increased compliance of our patients $(71 \%$ in the exercise group completed the programme), and suggests that centre-based exercise programmes for the rehabilitation of haemodialysis patients are preferred.

There were some limitations to our study. First, due to difficulties in recruiting patients for the morning shift, our sample size was small and lacked randomisation. Hence, it may not be possible to generalise our study findings. Second, as transportation was often a problem, many patients were unable to fully participate in the exercise sessions. Lastly, we did not investigate other health-related variables such as depression and the physical status of patients, which may have had a bearing on the results of our study. We suggest that future studies on the rehabilitation of haemodialysis patients assess the impact of exercise on their overall quality of life while taking into account all the aforementioned factors.

In conclusion, fatigue and quality of sleep were found to highly impact health-related quality of life in long-term haemodialysis patients. In our cohort of dialysis patients, a lowto-moderate-intensity exercise programme improved fatigue and sleep quality, which brought about an overall improvement in their quality of life post-treatment, thus suggesting the importance of exercise among dialysis patients. We recommend that centrebased exercise programmes be implemented for long-term haemodialysis patients in dialysis centres. Close collaboration and partnership between all stakeholders and healthcare personnel would be necessary to ensure the success of such programmes. Routine assessment of health-related quality of life should also be regularly conducted. Individual care plans and timely referrals to the appropriate teams are also pivotal. Such exercise facilities should become an integral part of the planning of future dialysis centres.

\section{ACKNOWLEDGEMENTS}

This study was supported by the Post Graduate Research Grant and University of Malaya Research Grant from the Health and Translational Medicine Cluster, University of Malaya, Malaysia. The authors are grateful to all the participants for their time and effort.

\section{REFERENCES}

1. Bonner $\mathrm{A}$, Wellard $\mathrm{S}, \mathrm{Caltabiano} \mathrm{M}$. The impact of fatigue on daily activity in people with chronic kidney disease. J Clin Nurs. 2010; 19:3006-15.

2. Li M, Li L, Fan X. Patients Having Hemodialysis: Physical Activity and Associated Factors. J Adv Nurs. 2010; 66:1338-45.

3. McCann K, Boore JRP. Fatigue in person with renal failure who require maintenance hemodialysis. J Adv Nurs. 2000; 32:1132-42.

4. O'Sullivan D, McCarthy G. An Exploration of relationship between fatigue and physical functioning in patients with end stage renal disease receiving hemodialysis. J Clin Nurs. 2007; 16:276-84.

5. Cupisiti A, Capitanini A, Betti G, D'Alessandro C, Barsotti G. Assessment of Habitual Physical Activity and Energy Expenditure in Dialysis Patients and Relationships to Nutritional Parameters. Clin Nephro. 2011; 75:3218-225.

6. Wong HS, Ong LM. Death And Survival On Dialysis: 18th Report of The Malaysian Dialysis and Transplant Registry.: The Malaysian Dialysis and Transplant Registry 2010.

7. Painter P, Carlson L, Carey S, Paul SM, Myll J. Physical Functioning and Health-Related Qulaity of Life Changes with Exercise Training in Hemodialysis Patients. Am J Kidney Dis. 2000; 35:482-92.

8. Stack AG, Murthy B. Exercise and Limitations in Physical Activity Levels among New Dialysis Patients in the United States: An Epidemiologic Study. Am J Epid. 2008; 18:880-8.

9. Stamatakis E, Hamer M. Sedentary Behavior: Redefining its Meaning and Links to Chronic Diseases. Brit J Hosp Med. 2011; 72:192-5.

10. Chang Y, Cheng S-Y, Lin M, Gau F-Y, C. Chao Y-F. The effectiveness of intradialytic leg ergometry exercise for improving sedentary life style and fatigue among patients with chronic kidney disease: A randomized clinical trial. Int J Nurs Stud. 2010; 47:1383-8.

11. Webster K, Cella D, Yost K. The functional assessment of chronic illness therapy (FACIT) measurement system: properties, applications and interpretation. Health Qual Life Outcomes. 2003; 1:1-7.

12. Borg GA. Psychophysical bases of percieved exertion. Med sci sports exerc. 1982; 14:377-81. 
13. Cella D, Lai JS, Chang $\mathrm{CH}$, Peterman A, Slavin M. Fatigue in cance patients compared with fatigue in general population. Am Ca Society. 2002; 94:528-38

14. Buysse DJ, Reynolds III CF, Monk TH, Berman SR, Kupfer DJ. The Pittsburgh sleep quality index: A new instrument for psychiatric practice and research. Psyc Res. 1989; 28:193-213.

15. Edmunds J, Ntoumanis N, Duda JL. A Test of Self-Determination Theory in the Exercise Domain. J Applied Soc Psy. 2006; 36:2240-65.

16. Lim YN, Ghazali A, Tan CC, Lee DG. Dialysis in Malaysia: 18th Report of The Malaysian Dilaysis and Transplant Registry 2010. Kuala Lumpur: Malaysia: Malaysian Society of Nephrology, 2011.

17. Karavatas SG, Tavakol K. Concurrent validity of borg's rating of perceived exertion in African-American young adults, employing heart rate as the standard. Internet J Allied Health Sciences Pract January 2005, Vol 3, No. 1

18. Yusop NB, Yoke MC, Shariff ZM, Beng HC. Factors Associated with Quality of Life among Hemodialysis Patients in Malaysia. PLoS ONE. 2013; 8:e84152.

19. Olson K. A New Way of Thinking about Fatigue: A Reconceptualization. Onco Nurs Forum. 2007; 34:93-9.

20. Santhana L, Srijit D, Hasliza H, et al. Fatigue Experienced by Patients Receiving Maintenance Dialysis in Hemodialysis Units. Nurs Health Sciences. 2011:60-4.
21. Merlino G, Piani A, Dolso P, et al. Sleep Disorders in patients with endstage renal disease undergoing dialysis therapy. Nephrol Dial Transp. 2005; 21:184-90.

22. Sklar AH, Riesenberg LA, Silber AK, Wagar A, Amir A. Postdialysis Fatigue. Am J Kidney Dis. 1996; 28:732-5.

23. Yurtkuran M, Alp A, Yutkuran M, Dilek K. A modified yoga-based exercise program in hemodialysis patints: a randomized controlled study. Comp Therapies Med. 2007; 65:164-71.

24. Malagoni AM, Catizane L, Mandini S, et al. Acute and long term effects of an exercise program for dialysis patients prescribed in hospital and performed at home. J Nephrol. 2008; 21:871-8.

25. Reza A, Abbas E, Nader S, Sanavi S. Effects of Interdialytic Aerobic Training on Sleep Quality in Hemodialysis Patients. J Kidney Dis. 2011; 5:119-23.

26. van Vilsteren MCBA, Greef MHGd, Huisman RM. The effects of a low-tomoderate intensity pre-conditioning exercise program linked with exercise counseling for sedentary haemodialysis patients in The Netherlands: results of a randomized clinical trial. Nephrol Dialysis Transp. 2004; 20:141-6.

27. Cheema BSB, Singh MAF. Exercise training in patients receiving maintenance hemodialysis: a systematic review of clinical trials. Am J Kidney Dis. 2005; 25:352- 64.

28. Ryan RM, Deci EL. Intrinsic and Extrinsic Motivations: Classic Definitions and New Directions. Contemp Edu Psycho. 2000; 25:54-67. 